\title{
Neuroimaging in Psychiatric Disorders
}

\author{
Joseph C. Masdeu \\ Section on Integrative Neuroimaging, Intramural Research Program, National Institutes of Health (NIH/NIMH-CBDB), 9000 \\ Rockville Pike, Building 10, Room 3C111, Bethesda, Maryland 20892-1365
}

Summary: In psychiatry, neuroimaging facilitates the diagnosis of psychiatric disorders and the development of new medications. It is used to detect structural lesions causing psychosis and to differentiate depression from neurodegenerative disorders or brain tumors. Functional neuroimaging, mostly in the form of molecular neuroimaging with positron emission tomography or single photon emission tomography, facilitates the identification of therapeutic targets, the determination of the dose of a new drug needed to occupy its target in the brain, and the selection of patients for clinical trials. Key Words: Magnetic resonance imaging, Positron emission tomography, Single photon emission tomography, Depression, Schizophrenia, Drug development.

\section{INTRODUCTION}

Unlike many neurological disorders, psychiatric disorders do not cause changes visible to the naked eye in the neuroimaging study of the individual patient [1]. They are, however, amenable to investigation by recent neuroimaging modalities, particularly quantitative structural imaging, as exemplified by voxel-based morphometry, and functional neuroimaging, using magnetic resonance imaging (MRI) techniques, positron emission tomography (PET), and single photon emission tomography (SPECT). Additionally, in the last few years psychiatric neuroimaging has benefitted from its interaction with genetics ("imaging genetics"). In such studies, neuroimaging findings in two or more genetic variants of the population are compared in order to discover imaging endophenotypes that may be amenable to measurement, and, therefore, useful as biomarkers in therapeutic discovery [2]. By relating neural structures to specific genes, imaging genetics is also likely to reveal the molecular underpinning of the organization and function of the various brain structures [2].

In this article I will review first the use of neuroimaging in the diagnosis of psychiatric disorders and then its use in the development of drugs to treat these disorders.

Electronic supplementary material The online version of this article (doi:10.1007/s13311-010-0006-0) contains supplementary material, which is available to authorized users.

Address correspondence and reprint requests to: Joseph C. Masdeu M.D., Ph. D. Section on Integrative Neuroimaging, Intramural Research Program, National Institutes of Health (NIH/NIMH-CBDB), 9000 Rockville Pike, Building 10, Room 3C111, Bethesda, MD 208921365. E-mail: masdeu@nih.gov.

\section{NEUROIMAGING IN THE DIFFERENTIAL DIAGNOSIS OF NEUROPSYCHIATRIC SYNDROMES}

Neuroimaging may help arrive at the correct diagnosis in a patient presenting with psychiatric symptoms. Such symptoms may be caused by neurological diseases masking as psychiatric disorders or by disorders currently considered to be primarily psychiatric in nature.

\section{Neuroimaging to diagnose neurological diseases masquerading as psychiatric disorders}

Neuroimaging is routinely used in the workup of patients with psychotic disorders because lesions of the frontal or temporal lobes, most often tumors, can present with psychosis [3]. In some cases, a behavioral syndrome is caused by focal seizures arising from a tumor, notably oligodendroglioma, or from congenital or traumatic lesions $[4,5]$. Psychotic features may also be found with thalamic or hypothalamic lesions $[6,7]$. Apathy caused by a frontal brain tumor may be mistaken for depression [8].

In older people with cognitive impairment, it may be difficult to differentiate a neurodegenerative disorder from depression. Neuroimaging may be helpful in this situation by showing findings characteristic of Alzheimer's disease (AD), diffuse Lewy body disease, or one of the frontotemporal dementias. AD is characterized by medial temporal atrophy on MRI or X-ray computed tomography (CT) [9], decreased metabolism in the parietotemporal association cortex, and precuneus on $\left[{ }^{18} \mathrm{~F}\right]$ fluoro-deoxyglucose (FDG) PET [9] and amyloid deposition on Pittsburgh compound B PET [10]. Many of 
these neuroimaging features are also present in diffuse Lewy body disease, but in addition to cognitive impairment patients have parkinsonian findings. The frontotemporal dementias, or other lobar dementia syndromes, can be identified early on FDG PET by decreased metabolism in the affected region, which eventually also shows atrophy on MRI (FIG. 1) [11].

In a patient with impairment in various areas of cognition, likely to be attributable to an attentional deficit, and with a normal result on the structural imaging study, a negative result on the PET or SPECT study lowers the probability of a neurodegenerative disorder [9, 12-14]. A normal result on the FDG PET study lowers the probability of the patient presenting a neurodegenerative disorder in the following few years to about $10 \%$ [13]. The $67 \%$ probability of a pathologically confirmed diagnosis of AD with a clinical diagnosis of possible AD drops to $52 \%$ with a negative result on the SPECT study [14]. A negative $\left[{ }^{11} \mathrm{C}\right]$-Pittsburgh compound B PET result will decrease the probability of $\mathrm{AD}$ and dementia with Lewy bodies (DLB), but not of having a frontotemporal dementia $[10,15]$. Very seldom will a neurologic disorder present with manic symptoms, although the disinhibition and behavioral disorders of the frontotemporal dementias of the Pick type may be confused with mania $[16,17]$.
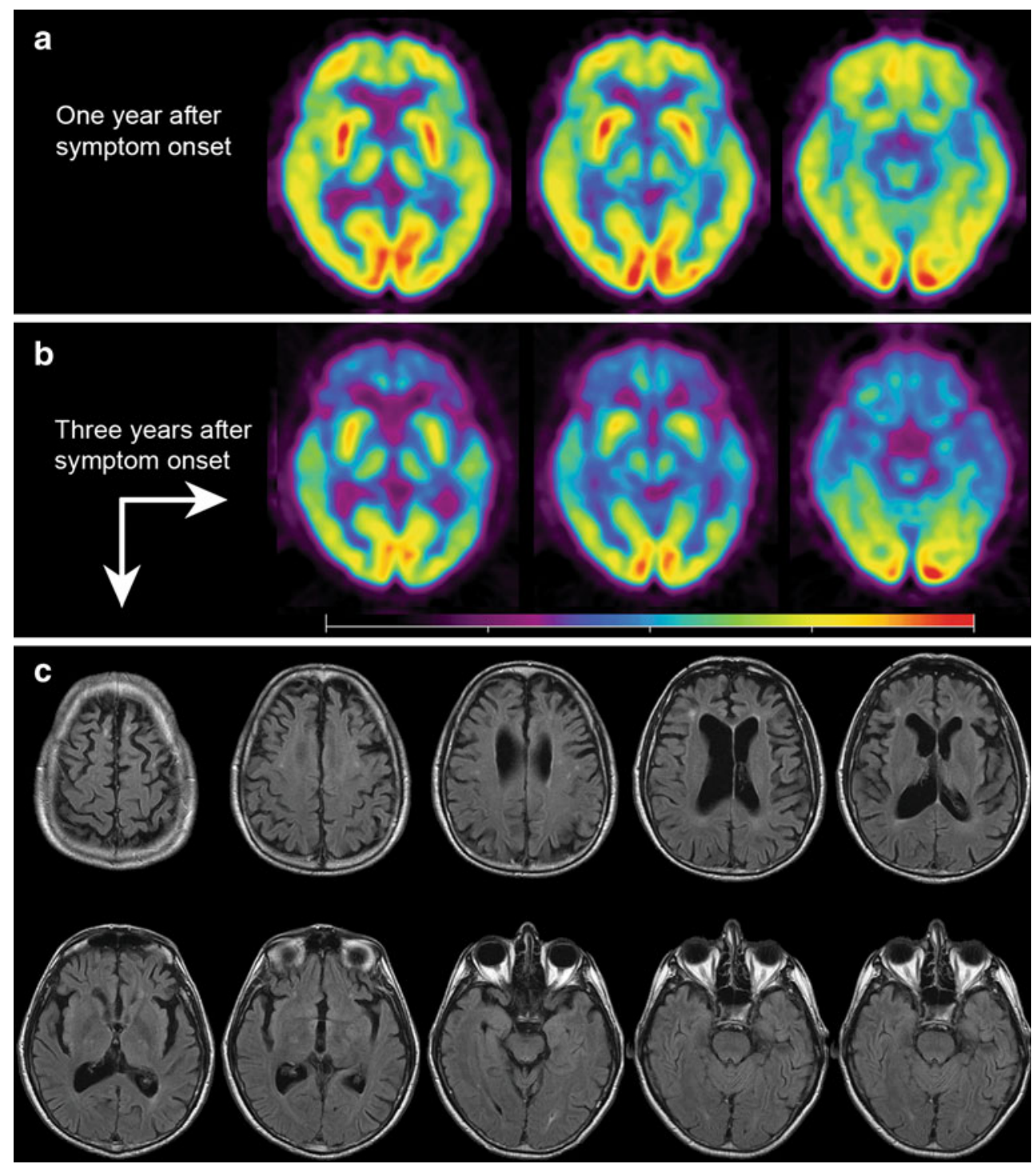

FIG. 1. Frontotemporal dementia. (a, b) FDG-PET and (c) FLAIR MRI studies from a 51-year-old man with progressive speech apraxia and impaired planning, to the point of mutism and complete dependency for activities of daily living when studies (b) and (c) were performed, on the same day. Metabolism was already decreased on the initial PET study, particularly on the frontal opercula and temporal tips, but it is much more obvious on the follow-up study, showing extensive frontotemporal hypometabolism. Note that the frontotemporal abnormality is much more obvious on the PET study (a, b) than on the MRI study (c), which shows frontal atrophy. FDG-PET $=\left[{ }^{18} \mathrm{~F}\right]$ fluoro-deoxyglucose positron emission tomography; FLAIR MRI = fluid-attenuated inversion-recovery magnetic resonance imaging. Reproduced with permission from Masdeu [57]. Copyright @ 2008, American Academy of Neurology. (High resolution version of this image is available in the electronic supplementary material.) 


\section{Neuroimaging in the diagnosis of psychiatric disorders}

Neuroimaging of psychiatric disorders has to contend with the diagnostic issue in psychiatry. Psychiatric diagnoses depend on an array of clinical manifestations that reflect neuronal network dysfunction [18]. However, network abnormalities may differ among individuals with the same diagnosis [19]. Moreover, similar network abnormalities could be caused by diverse etiologies. For these reasons, the neurobiology of psychiatric disorders is likely to be highly heterogeneous. Even when for clinical and research purposes entities such as schizophrenia and depression are used as if they were a single disease, these disorders are more likely to be syndromic groupings of an array of different diseases. No surprise then that the neuroimaging findings in psychiatric disease may lack specificity and often fail to reveal a clear connection to a single neurobiological disturbance. Currently, the neuroimaging pattern determined in the study of a single psychiatric patient does not allow for an accurate diagnosis. Some characteristic findings, however, have been derived from samples of patients with each of the psychiatric diagnostic groupings. Only those that have been duplicated by several groups will be mentioned here.

Schizophrenia. Based on results from voxel-based morphometry or other quantitative structural MRI techniques, the volume of the prefrontal, anterior temporal, and perisylvian regions, as well as of the anteromedial thalamus, has been found to be decreased even in first time psychotic episodes [20, 21]. However, similar structural changes can be effected by the use of antipsychotic medication [22]. Functional MRI (fMRI) or $\mathrm{H}_{2}{ }^{15} \mathrm{O}$ PET scans have revealed abnormal activation in the prefrontal and cingulate cortex and the medial temporal lobe $[23,24]$. The interaction between these structures is also abnormal $[25,26]$. Decreased suppression of the restingstate brain network during stimulation paradigms has also been reported in schizophrenia [27]. Abnormalities in dopaminergic activity will be reviewed in the section on the application of neuroimaging to drug development.

Major depressive disorder. By contrast with some of the neurodegenerative disorders that can mimic depression and which are accompanied by decreased frontal lobe metabolism, in depression, elevated metabolism but reduced volume have been found in the subgenual region of the medial frontal lobe [28]. Also, the activation pattern on fMRI has been found to distinguish these disorders. Hippocampal activation during a memory task was found to be decreased in AD patients compared with controls and depressed patients [29]. In contrast, orbitofrontal and cingulate activation were greater in depressed patients than in $\mathrm{AD}$ subjects or healthy controls [29]. Genotypic variants are likely to influence both the likelihood of developing major depression and the imaging findings. For instance, in depression, increased activity of the amygdala in response to negative stimuli appears to be modulated by the 5-HT transporter gene (SLC6A4) promoter polymorphism (5-HTTLPR) [28]. Hippocampal volume loss is characteristic of elderly or chronically ill and depressed individuals and may be impacted by the val66met brain-derived neurotrophic factor gene variant and the 5-HTTLPR SLC6A4 polymorphism. In terms of neuroreceptor PET imaging, major depression has been associated with decreased 5-HT(1A) binding potential in the raphe nuclei, medial temporal lobe, and medial prefrontal cortex [28].

\section{NEUROIMAGING IN DRUG DISCOVERY AND DEVELOPMENT}

This section draws heavily from the excellent review by Wong et al. (2009) [30]. Neuroimaging can be helpful at several levels of drug discovery and development: 1) in characterizing preclinical models; 2) in early clinical studies to show that target engagement by the new drug induces the biological changes expected to give clinical benefit; 3) in clinical trials to demonstrate proof of concept (PoC) or, in other words, that engaging a particular target is linked to a meaningful change in a clinical endpoint and thus proving the effectiveness of the drug being tried. An example of the use of neuroimaging in preclinical models is the demonstration that a new PET compound, $\left[{ }^{11} \mathrm{C}\right]$ GSK 931145 , binds the glycine transporter 1, an important modulator of NMDA receptors, which are considered to be hypoactive in schizophrenia [31, 32]. The brain distribution of this compound can then be studied in the rat, larger experimental animals, and humans (FIG. 2). A comparison of the brain distribution of the glycine transporter in schizophrenic patients and healthy controls based on the presence of this compound could allow for the determination of a potential role of the glycine transporter 1 in schizophrenia. If this transporter were found to have abnormal activity in schizophrenia, drugs could be designed to return its activity to normal. The biological efficacy of such drugs could then be monitored with neuroimaging: displacement of $\left[{ }^{11} \mathrm{C}\right]$ GSK931145 would mean that the experimental drug is binding the glycine transporter 1 [32]. Still, the abnormal function of this potential drug target may not play a role in the disease but, rather, be a byproduct of other deranged mechanisms. For this reason, a clinical trial is still needed to prove that the new drug is beneficial in schizophrenia.

Neuroimaging provides a window to observe "in vivo" brain structure and function, both of which are likely to 

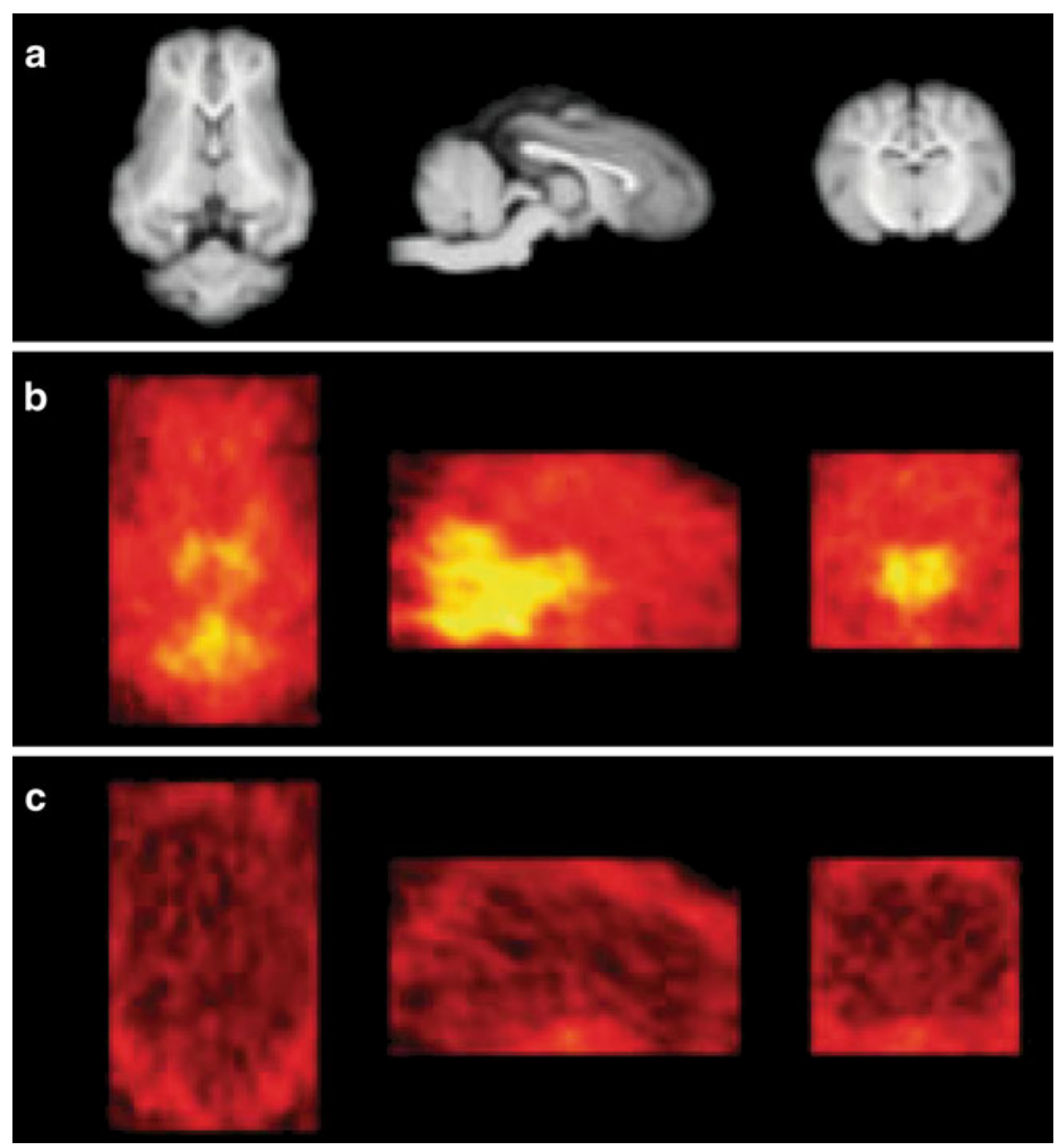

FIG. 2. New radioligand for the glycine transporter 1. Transaxial, sagittal, and coronal images of the MRI (a) and PET (b, c) in an experimental animal (pig). The PET image was obtained using the new radioligand $\left[{ }^{11} \mathrm{C}\right] \mathrm{GSK} 931145$, which binds to regions known to contain abundant transporter, mainly in the brainstem, cerebellum, and thalamus. To demonstrate the specificity of the radioligand, binding of the radioligand in (c) has been blocked by the administration of a potent glycine transporter 1 inhibitor. Reproduced with permission from Passchier et al. [31]. Copyright () 2010, John Wiley and Sons. (High resolution version of this image is available in the electronic supplementary material.)

be abnormal in two of the commonest disorders affecting humankind: depression and schizophrenia. As such, it could be an ideal biomarker in therapeutic drug development. A biomarker is defined as a characteristic that can be objectively measured and evaluated as an indicator of normal or abnormal biologic processes, or as an indicator of pharmacological responses to a therapeutic intervention [33]. In a progression from less to more specificity, biomarkers have been classified into three types: type 0 is used to track the natural course of the disease; type 1 can be used to examine the effects of intervention along with the known mechanism of action of the drug but without a strict relationship to clinical outcome; finally, a change in type 2 biomarkers is predictive of clinical outcome [30]. At present, most imaging methods in psychiatry do not meet the biomarker status. Some, however, can be considered to be emerging biomarkers or pre-biomarkers because they allow for the identification of therapyrelevant characteristics of the disease. For instance, by imaging striatal dopamine (DA) D2 receptors using $\left[{ }^{11} \mathrm{C}\right]$ raclopride $\mathrm{PET}$, a link was found between $\mathrm{D} 2$ receptor occupancy by medication and a reduction in positive symptoms in schizophrenia: ascending doses of up to $80 \%$ receptor occupancy were progressively more effective in relieving delusions and hallucinations [34]. This result also explained some of the clinical side effects of higher doses: above an $80 \%$ occupancy there was no therapeutic benefit, only an increase in extrapyramidal side effects, including akathisia. These findings make D2 PET a useful tool to strengthen the PoC of a given drug being effective for positive symptoms: if it blocks D2 striatal receptors, it is likely to work [30]. Additionally, as receptor occupancy can be measured, PET is a useful tool by which to establish a dose range when testing for new, pure D2 antagonists. However, antipsychotic activity may not depend on the blockade of striatal D2 binding, but rather on the blockade of extra-striatal binding sites. Atypical antipsychotics, such as clozapine, which are effective in alleviating positive symptoms while producing minimal parkinsonian sideeffects, block preferentially D2 receptors in regions outside the striatum, such as the temporal lobe $[35,36]$.

In general, neuroimaging can help in a number of the steps needed to determine the properties of a candidate 
drug. It is critical to determine first whether the drug crosses the blood-brain barrier (BBB) and thus is delivered to the target compartment, namely, the brain. The speed of delivery and possible competition with other substances are included in what are termed the pharmacokinetic properties of the drug in development. Next, it is important to determine if the drug engages the appropriate target, be it a receptor, a transporter system, or an enzyme, and whether it does so in a dose/exposurerelated manner. After it has engaged the target, it is critical to determine whether it causes the biological effects expected from target activation [30]; these are the pharmacodynamic properties of the drug. The following sections present examples of how neuroimaging is being used to obtain information on the various steps of candidate drug behavior.

\section{Role of various neuroimaging modalities in drug development}

PET and SPECT. Both PET and SPECT have been used successfully to explore a number of neurotransmitter systems. Best studied as regards drug development in psychiatry is the dopaminergic system. In terms of PET tracers, presynaptic DA synthesis and storage have been studied with $\left[{ }^{18} \mathrm{~F}\right]$ fluoro-DOPA [37]; postsynaptic D1,5 receptor binding has been studied with $\left[{ }^{11} \mathrm{C}\right] \mathrm{NNC} 112$ [38]; striatal postsynaptic D2,3,4 receptor binding, of great interest in psychiatry, has been measured with $\left[{ }^{11} \mathrm{C}\right]$ raclopride [39]. Newer compounds with greater affinity are currently being used to study D2,3,4 receptors in the entire brain, including the cortex. The most widely used of these are $\left[{ }^{11} \mathrm{C}\right] \mathrm{FLB} 457$ and $\left[{ }^{18} \mathrm{~F}\right]$ fallypride $[40,41]$. As ${ }^{18} \mathrm{~F}$ has a much larger half life $(110 \mathrm{~min})$ than ${ }^{11} \mathrm{C}(20 \mathrm{~min}),{ }^{18} \mathrm{~F}$ is easier to use and allows for scanning in facilities that have a PET camera, but not a cyclotron. The high binding affinity of fallypride requires relatively long studies that last for at least $3.5 \mathrm{~h}$.

Several analogues of cocaine have been investigated to develop DA transporter selective radioligands for SPECT imaging, of which $\left[{ }^{123} \mathrm{I}\right] \beta$-CIT and $\left[{ }^{123} \mathrm{I}\right] \mathrm{Ioflupane}\left(\left[{ }^{123} \mathrm{I}\right]\right.$ FP-CIT) are the most widely used. The main advantage of $\left[{ }^{123} \mathrm{I}\right]$ Ioflupane is that images can be acquired from 3 to $6 \mathrm{~h}$ postinjection, compared with the 18-24 $\mathrm{h}$ required for $\left[{ }^{123} \mathrm{I}\right] \beta$-CIT [42]. A D2 receptor marker $\left(\left[{ }^{123} \mathrm{I}\right] \mathrm{IBZM}\right)$ is also commercially available in Europe and has been used in the USA for clinical studies of schizophrenia [43, 44].

If the study requires anatomical detail, in addition to the relatively low-resolution PET ( $\sim 7 \mathrm{~mm}$ resolution) or SPECT $(\sim 10 \mathrm{~mm}$ resolution) images, high-resolution $(\sim 1-2 \mathrm{~mm})$ structural images can be obtained with MRI [45]. Functional and structural images of the same subject are co-registered to yield anatomically detailed images.
In untreated schizophrenia there is decreased D2 receptor binding in the dorsomedial nucleus of the thalamus, striatum, anterior cingulate cortex, amygdala, and temporal cortex [46]. This reduced binding may be explained by a greater receptor saturation or even by downregulation of the receptor, induced by an increased secretion of DA in these regions, and has also been documented in schizophrenia by means of $\left[{ }^{18} \mathrm{~F}\right]$ fluoroDOPA PET [37]. Still, the net effect may be that of increased D2 receptor stimulation, such that blockage of D2 receptors by neuroleptics is therapeutic.

PET has been used for a variety of applications in drug development, for instance, by using established or newly developed PET radiotracers for a particular target. Targets being sought depend on the neurobiology of the disease: DA is a likely target for schizophrenia; serotonin is a likely target for depression. For this reason, PET ligands have been developed to assess serotonin receptors and transporters in this disorder. Secondly, PET has been used to determine the degree of target engagement needed to exert therapeutic effects, for instance, by determining norepinephrine transporter occupancy in attention-deficit hyperactivity disorder or cannabinoid occupancy in obesity and other psychiatric disorders [30]. Thirdly, PET can be used to study the effect of an enzyme or a second messenger system, such as phosphodiesterases $\mathrm{I}-\mathrm{V}$ in depression [30].

Two major approaches have been used in PET drug development: 1) to radiolabel the new drug and 2) to estimate its target occupancy [30]. Once the new drug has been radiolabeled, important characteristics can be determined, such as its brain distribution, some of its washout characteristics, and whether it is a substrate for BBB pumps. Depending on the label, studies can be carried out not only on experimental animals, but in humans as well. However, the radiolabeling of candidate drugs is not a straightforward procedure, and primary quantitative radiotracers label only a very small number of potential drugs. In addition, the interpretation of the findings with available labeled drugs requires care. Given the short acquisition times possible with these isotopes (maximum of $90 \mathrm{~min}$ with ${ }^{11} \mathrm{C}$ and $8 \mathrm{~h}$ for ${ }^{18} \mathrm{~F}$ ) and even the shorter standard scanning times, the brain exposure for compounds that cross the BBB slowly may be underestimated. Higher brain concentrations after chronic dosing may also lead to an underestimation of brain exposure when the study is performed during early dosing. By contrast, true target engagement may be overestimated with PET. This is the case when the total regional brain activity is recorded, and binding to the test drug is not separated from radiolabeled metabolites of the test drug and free tracer and tracer nonspecific binding [30].

The difficulties experienced in labeling new drugs and measuring accurately their brain distribution have led to a 
different strategy for many drug development studies using PET or SPECT: the target is labeled instead of the drug. For this purpose, existing PET/SPECT tracers are used to determine the target occupancy of the new drug in displacement studies. A more active drug translates into less labeled target being available. For instance, in one study, the serotonin transporter was labeled with 123I-ADAM SPECT; brain distribution of the tracer was found to be greatest in the medial raphe region of pons and midbrain, providing anatomical evidence of the specificity of the tracer [47]. Both depressed patients and healthy controls had similar levels of the tracer. After a 6-week course of paroxetine $(20 \mathrm{mg} /$ day $)$, a $71 \%$ decrease in serotonin transporter occupancy was observed in the midbrain (FIG. 3).

Whether to use PET or SPECT for molecular functional studies depends on the availability of radiotracers and on the need for anatomical resolution. For studies in humans, PET currently has a greater anatomical resolution than SPECT. However, for small animal studies, SPECT cameras, in units combined with X-ray $\mathrm{CT}$, are available which have greater spatial resolution than PET [48]. Catafau et al. [39] compared the two nuclear medicine techniques in a study of D2 receptor occupancy. The same subjects were given [123I]IBZM, a SPECT tracer, and $\left[{ }^{11} \mathrm{C}\right]$ Raclopride, a PET tracer. Occupancy values measured by SPECT (12.4\%, using occipital cortex as reference region) were lower than those measured with PET (13.8\%, using the cerebellum as reference region). Anatomical resolution was superior in the PET studies. However, there was an almost perfect correlation between D2 occupancy in individual subjects using either method [39].

Drug binding characteristics have a major effect on drug evaluation by PET. Neutral orthosteric site antagonists, such as haloperidol, are relatively straightforward to assess by means of PET. However, considerable challenges exist in the assessment of target engagement and in linking it to efficacy for agonists, partial agonists, such as aripiprazole, inverse agonists, and allosteric modulators [30]. Many new therapeutic approaches to psychiatric disorders use positive allosteric modulators as a means to fine-tune the primary excitatory (Glu) and inhibitory [ $\gamma$-aminobutyric acid (GABA)] systems. Aripiprazole provides an example of the caution to be used interpreting drug behavior with PET. As mentioned, the original PET studies with neuroleptics showed that DA D2 antagonists have antipsychotic efficacy with minimal extrapyramidal syndrome side effects within a 'therapeutic window' of $65-80 \%$ striatal D2 receptor occupancy. However, for aripiprazole, occupancies closer to 90 or $95 \%$ were needed for the therapeutic range of the drug [49]. This behavior on PET reflects the likely mechanism of action of aripiprazole, which is a partial
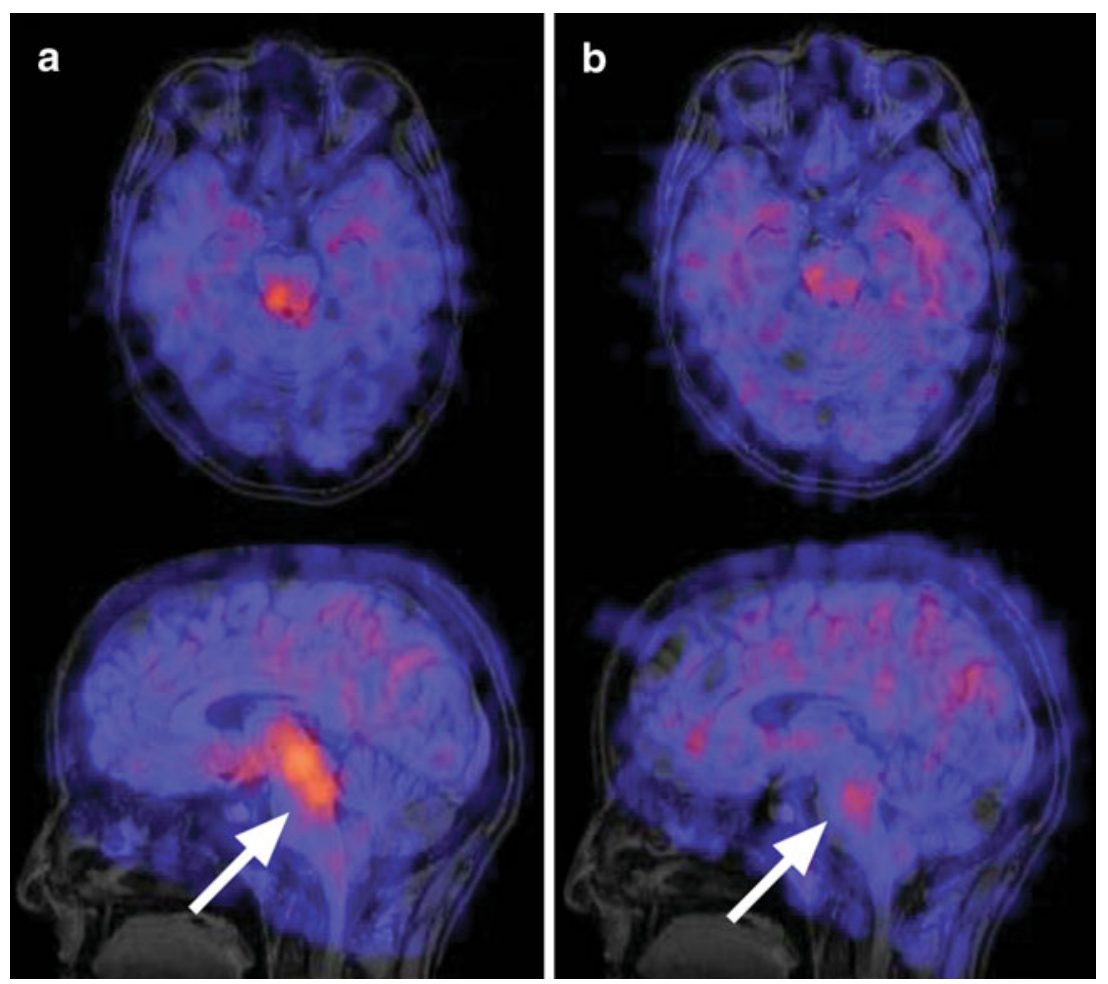

FIG. 3. SERT occupancy by paroxetine. Coregistered MRI and $\left[{ }^{123} \mid\right] A D A M ~ S P E C T$ images from a patient with major depression at baseline (a) and after a 6-week treatment with paroxetine $20 \mathrm{mg} /$ day (B). Note the decreased uptake in the medial raphe region (arrow) in the second scan (b), both on axial (top) and sagittal slices (bottom), corresponding to 71\% SERT occupancy. Reprinted with permission from Catafau et al. [47]. Copyright (C) 2006, Springer Science+Business Media. SERT = Serotonin transporter; SPECT = single photon emission tomography. (High resolution version of this image is available in the electronic supplementary material.) 
agonist at D2 receptors. The original "therapeutic window" of $65-80 \%$ receptor occupancy is valid only for DA D2 antagonists [30].

Similarly, when studying dosing for antidepressants, serotonin reuptake inhibitors (SSRIs) have been shown to occupy $\geq 80 \%$ of the serotonin transporter (SERT) at clinically used doses; within this class of drugs, this occupation seems to be independent of the specific SSRI. However, the tricyclic antidepressant (TCA) clomipramine occupies $80 \%$ of the SERT at doses as low as $10 \mathrm{mg}$, at a plasma concentration of $1.42 \mathrm{ng} / \mathrm{ml}$ [50]. Yet, clinically used doses are $50-150 \mathrm{mg}$ per day and therapeutic plasma concentrations range between 175 and $450 \mathrm{ng} / \mathrm{ml}$ [51]. This finding leads to some obvious questions [30]: Is blockade of the SERT and the norepinephrine transporter the therapeutic principle of clomipramine (and of the TCAs in general)? Or do TCAs behave completely different from SSRIs due to their broad pharmacological actions at many different molecular targets? How valid are the studies upon which therapeutic doses and plasma concentrations have been determined for clinical use of the TCAs over decades? Some of the answers to these questions rest on the pharmacology of the various compounds [30]. Agonists and partial agonists present a complex pharmacology for PET occupancy studies. Agonists require only a very partial occupancy to exert their pharmacological effects. For instance, occupancy of the GABAA-benzodiazepine receptor site by benzodiazepines is only $15-25 \%$ at full pharmacological effect. These compounds act with a very high receptor reserve. Therefore, when using PET to calculate dosing, it is important to remember that a radiotracer/pre-biomarker that has been demonstrated to predict the biological effects of a certain class of compounds might lose its validity for a drug with a slight modification of its mechanism of action even if it binds to the same target molecule [30].

Another application of PET imaging in dose finding is in the early rejection of new drugs. If a dose that demonstrated adequate target engagement in humans does not have efficacy in the clinical trial, $\mathrm{PoC}$ can be rejected, and a drug target can be abandoned more quickly. For instance, $\left[{ }^{18} \mathrm{~F}\right] \mathrm{SPA}-\mathrm{RQ}$ PET showed how the drug aprepitant blocked its target, the neurokinin 1 receptor. However, it was no better than placebo in terms of improving clinical depression as measured with the Hamilton Depression Scale (FIG. 4) [52]. PET imaging demonstrated that the drug was ineffective even when the dose given was sufficient to adequately engage its target.

fMRI. While fMRI is much more widely used than PET for the study of cognition, it has not been used as much in drug development. However, this technique has the potential to show the mending of abnormal brain function with effective new medications. For example, fMRI has been used as a pre-biomarker in the study of depression. The SSRI antidepressant citalopram reduced amygdala activation in response to fearful faces in normal volunteers [53]. The amygdala response to fearful stimuli could develop into a pre-biomarker for antidepressant effects.

Magnetic resonance spectroscopy. This technique has been applied to the assessment of the mechanism of action of psychotropic drugs with GABAergic or glutamatergic mechanisms of action, by measuring "in vivo" glutamate and GABA amino acids. For instance,
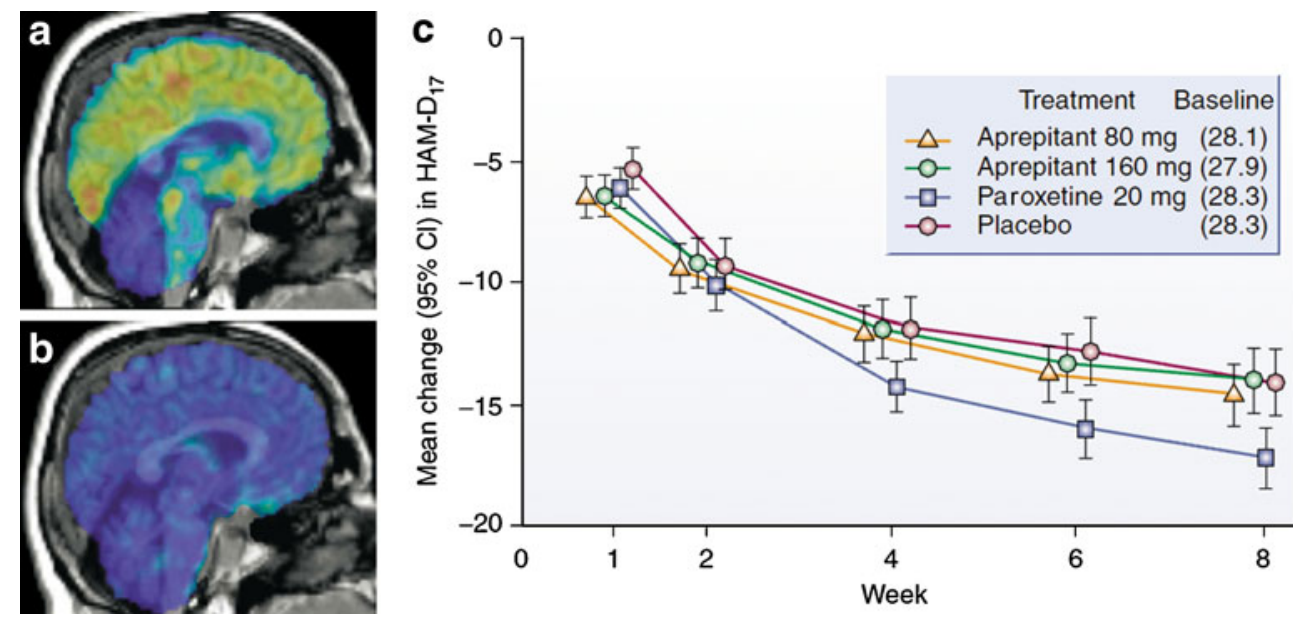

FIG. 4. Early rejection of drug target. ( $a$ and $b$ ) In these PET studies, overlaid on the MRI of the same patient, neurokinin-1 receptors are visualized with $\left[{ }^{18} \mathrm{~F}\right] \mathrm{SPA}-\mathrm{RQ}$ before (a) and after (b) blockade by the administration of $160 \mathrm{mg}$ of aprepitant for 40 days. The warmer yelloworange tones (a) indicate greater binding of the PET compound, whereas the blue tones (b) indicate little binding of the receptor, now occupied by aprepitant. (c) Graphic of the evolution of scores on the Hamilton Depression Scale (y-axis) over 8 weeks ( $x$-axis) with the intake of two different doses of aprepitant, the antidepressant paroxetine, and a placebo. Despite the occupancy of the neurokinin-1 receptor by aprepitant, medication with this drug has similar efficacy to placebo in improving depression. Reprinted with permission from Kessler et al. [52] Panel C is modified from Wong et al. [30]; reprinted by permission from Macmillan Publishers @ 2009. (High resolution version of this image is available in the electronic supplementary material.) 
the improvement of bipolar depression with cytidine has been linked to decreased glutamate levels in the anterior cingulate region (FIG. 5) [54].

Diffusion tensor imaging. Diffusion tensor imaging has been used to determine white matter integrity in patients with depression who have undergone multiple treatments with transcranial magnetic stimulation, as a means of proving that this treatment is safe even if applied repeatedly [55]. Using tractography, Coenen et al. [56] documented in patients being treated for Parkinson's disease with subthalamic stimulation that those patients who developed mania with stimulation had electrodes placed in the proximity of the medial forebrain bundle. This
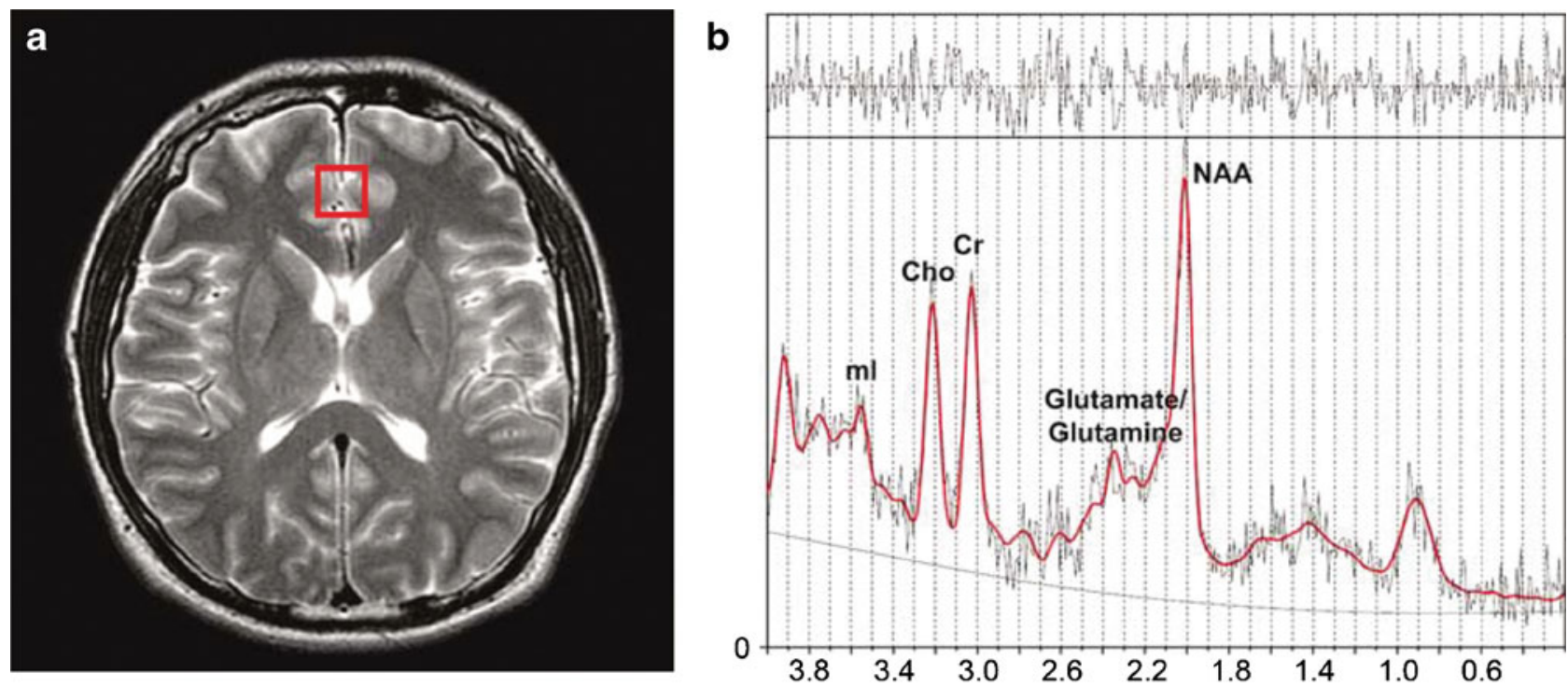

C

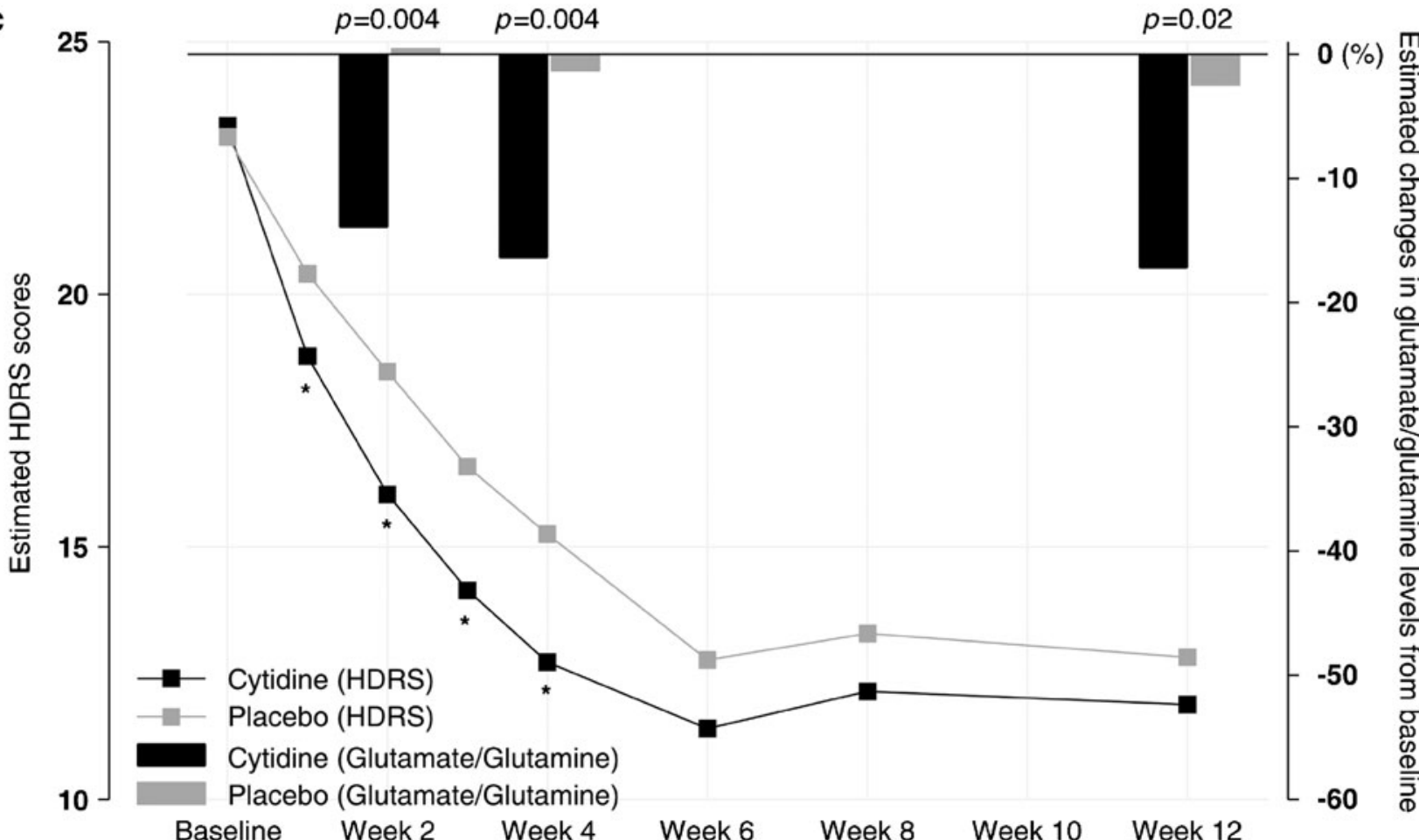

FIG. 5. Mechanism of action of cytidine in depression. (a) Cubic volume in the anterior cingulate cortex where MR spectroscopy was performed to obtain the spectrum shown in (b), with identifiable glutamate peaks. The graphic in (c) shows how the cytidine-treated group has a significantly better outcome than placebo on the HDRS, as well as a greater decrease in glutamate levels in the anterior cingulate region. HDRS $=$ Hamilton Depression Rating Scale; NAA = N-acetylaspartate; Glx = glutamate/glutamine; $\mathrm{Cr}=$ creatine; Cho = choline; $\mathrm{ml}=$ myo-inositol. Reprinted by permission from Yoon et al. [54]. Copyright (C) 2009, Macmillan Publishers. (High resolution version of this image is available in the electronic supplementary material.) 
finding has potential for the development of depression treatment strategies based on deep brain stimulation.

\section{CONCLUSION}

In summary, neuroimaging is being increasingly applied in the development of psychiatric therapies. Neuroimaging enables the clinician to test whether a potential drug target is abnormal in a psychiatric disorder and whether, therefore, its correction may be therapeutic. By labeling a new drug or its target, BBB penetration and target binding can be assessed, as well as the dosing needed to accomplish these. Finally, by showing which individuals manifest the expected pharmacological action of the drug, neuroimaging allows for the selection of the group of individual most likely to benefit in a clinical trial.

Acknowledgments: This work was supported by the Intramural Research Program of the National Institute of Mental Health, National Institutes of Health, USA.

\section{REFERENCES}

1. Agarwal N, Port JD, Bazzocchi M, Renshaw PF. Update on the use of MR for assessment and diagnosis of psychiatric diseases. Radiology 2010;255:23-41.

2. Inkster B, Nichols TE, Saemann PG, et al. Pathway-based approaches to imaging genetics association studies: Wnt signaling, GSK3beta substrates and major depression. Neuroimage 2010;53: 908-917.

3. Bunevicius A, Deltuva VP, Deltuviene D, Tamasauskas A, Bunevicius R. Brain lesions manifesting as psychiatric disorders: eight cases. CNS Spectr 2008;13:950-958.

4. Sherwin I, Peron-Magnan P, Bancaud J, Bonis A, Talairach J. Prevalence of psychosis in epilepsy as a function of the laterality of the epileptogenic lesion. Arch Neurol 1982;39:621-625.

5. Boone KB, Miller BL, Rosenberg L, Durazo A, McIntyre H, Weil M. Neuropsychological and behavioral abnormalities in an adolescent with frontal lobe seizures. Neurology 1988;38:583586.

6. Moise D, Madhusoodanan S. Psychiatric symptoms associated with brain tumors: a clinical enigma. CNS Spectr 2006;11:28-31.

7. Albert DJ, Walsh ML, Jonik RH. Aggression in humans: what is its biological foundation? Neurosci Biobehav Rev 1993;17:405-425.

8. Grafman J, Vance SC, Weingartner H, Salazar AM, Amin D. The effects of lateralized frontal lesions on mood regulation. Brain 1986;109:1127-1148.

9. Masdeu JC, Zubieta JL, Arbizu J. Neuroimaging as a marker of the onset and progression of Alzheimer's disease. J Neurol Sci 2005;236:55-64.

10. Rowe $\mathrm{CC}, \mathrm{Ng} \mathrm{S}$, Ackermann U, et al. Imaging beta-amyloid burden in aging and dementia. Neurology 2007;68:1718-1725.

11. Foster NL, Heidebrink JL, Clark CM, et al. FDG-PET improves accuracy in distinguishing frontotemporal dementia and Alzheimer's disease. Brain 2007;130:2616-2635.

12. Jagust W, Reed B, Mungas D, Ellis W, Decarli C. What does fluorodeoxyglucose PET imaging add to a clinical diagnosis of dementia? Neurology 2007;69:871-877.

13. Silverman DH, Small GW, Chang CY, et al. Positron emission tomography in evaluation of dementia: Regional brain metabolism and long-term outcome. JAMA 2001;286:2120-2127.

14. Jagust W, Thisted R, Devous MD, Sr., et al. SPECT perfusion imaging in the diagnosis of Alzheimer's disease: a clinicalpathologic study. Neurology 2001;56:950-956.
15. Lowe VJ, Kemp BJ, Jack CR, Jr., et al. Comparison of 18F-FDG and PiB PET in cognitive impairment. J Nucl Med 2009;50:878886.

16. Reisch T, Brekenfeld C, Barth A. A case of hydrocephalus occlusus presenting as bipolar disorder. Acta Psychiatr Scand 2005;112:159162; discussion 162-153.

17. McMurtray AM, Chen AK, Shapira JS, et al. Variations in regional SPECT hypoperfusion and clinical features in frontotemporal dementia. Neurology 2006;66:517-522.

18. American Psychiatric Association. Diagnostic and statistical manual of mental disorders (DSM-IV-TR). Arlington, Va: American Psychiatric Association; 2000.

19. Markou A, Chiamulera C, Geyer MA, Tricklebank M, Steckler T. Removing obstacles in neuroscience drug discovery: the future path for animal models. Neuropsychopharmacology 2009;34:74-89.

20. Sun D, van Erp TG, Thompson PM, et al. Elucidating a magnetic resonance imaging-based neuroanatomic biomarker for psychosis: classification analysis using probabilistic brain atlas and machine learning algorithms. Biol Psychiatry 2009;66:1055-1060.

21. Meisenzahl EM, Koutsouleris N, Bottlender R, et al. Structural brain alterations at different stages of schizophrenia: a voxel-based morphometric study. Schizophr Res 2008;104:44-60.

22. Thompson PM, Bartzokis G, Hayashi KM, et al. Time-lapse mapping of cortical changes in schizophrenia with different treatments. Cereb Cortex 2009;19:1107-1123.

23. Eisenberg DP, Sarpal D, Kohn PD, et al. Catechol-o-methyltransferase valine(158)methionine genotype and resting regional cerebral blood flow in medication-free patients with schizophrenia. Biol Psychiatry 2010;67:287-290.

24. Blasi G, Taurisano P, Papazacharias A, et al. Nonlinear response of the anterior cingulate and prefrontal cortex in schizophrenia as a function of variable attentional control. Cereb Cortex 2010;20:837845.

25. Meyer-Lindenberg AS, Olsen RK, Kohn PD, et al. Regionally specific disturbance of dorsolateral prefrontal-hippocampal functional connectivity in schizophrenia. Arch Gen Psychiatry 2005;62:379-386.

26. Benetti S, Mechelli A, Picchioni M, Broome M, Williams S, McGuire P. Functional integration between the posterior hippocampus and prefrontal cortex is impaired in both first episode schizophrenia and the at risk mental state. Brain 2009;132:24262436.

27. Whitfield-Gabrieli S, Thermenos HW, Milanovic S, et al. Hyperactivity and hyperconnectivity of the default network in schizophrenia and in first-degree relatives of persons with schizophrenia. Proc Natl Acad Sci USA 2009;106:1279-1284.

28. Savitz JB, Drevets WC. Imaging phenotypes of major depressive disorder: genetic correlates. Neuroscience 2009;164:300-330.

29. Gron G, Bittner D, Schmitz B, Wunderlich AP, Riepe MW. Subjective memory complaints: objective neural markers in patients with Alzheimer's disease and major depressive disorder. Ann Neurol 2002;51:491-498.

30. Wong DF, Tauscher J, Grunder G. The role of imaging in proof of concept for CNS drug discovery and development. Neuropsychopharmacology 2009;34:187-203.

31. Passchier J, Gentile G, Porter R, et al. Identification and evaluation of [11C]GSK931145 as a novel ligand for imaging the type 1 glycine transporter with positron emission tomography. Synapse 2010;64:542-549.

32. Murthy N, Passchier J, Gunn RN, et al. $\left[{ }^{11} \mathrm{C}\right]$ GSK931145: A new PET ligand for glycine transporter 1. Neuro-Receptor Meeting. Pittsburgh 2008.

33. Lesko LJ, Atkinson AJ, Jr. Use of biomarkers and surrogate endpoints in drug development and regulatory decision making: criteria, validation, strategies. Annu Rev Pharmacol Toxicol 2001;41:347-366.

34. Kapur S, Zipursky R, Jones C, Remington G, Houle S. Relationship between dopamine $\mathrm{D}(2)$ occupancy, clinical response, and side effects: a double-blind PET study of first-episode schizophrenia. Am J Psychiatry 2000;157:514-520.

35. Grunder G, Landvogt C, Vernaleken I, et al. The striatal and extrastriatal $\mathrm{D} 2 / \mathrm{D} 3$ receptor-binding profile of clozapine in patients with schizophrenia. Neuropsychopharmacology 2006;31:10271035 . 
36. Kessler RM, Ansari MS, Riccardi P, et al. Occupancy of striatal and extrastriatal dopamine D2 receptors by clozapine and quetiapine. Neuropsychopharmacology 2006;31:1991-2001.

37. Meyer-Lindenberg A, Miletich RS, Kohn PD, et al. Reduced prefrontal activity predicts exaggerated striatal dopaminergic function in schizophrenia. Nat Neurosci 2002;5:267-271.

38. Slifstein M, Kolachana B, Simpson EH, et al. COMT genotype predicts cortical-limbic D1 receptor availability measured with [(11)C]NNC112 and PET. Mol Psychiatry 2008;13:821-827.

39. Catafau AM, Suarez M, Bullich S, et al. Within-subject comparison of striatal D2 receptor occupancy measurements using [123I]IBZM SPECT and [11C]Raclopride PET. Neuroimage 2009;46:447-458.

40. Kessler RM, Woodward ND, Riccardi P, et al. Dopamine D(2) receptor levels in striatum, thalamus, substantia nigra, limbic regions, and cortex in schizophrenic subjects. Biol Psychiatry 2009;65:1024-1031.

41. Kegeles LS, Slifstein M, Frankle WG, et al. Dose-occupancy study of striatal and extrastriatal dopamine $\mathrm{D}(2)$ receptors by aripiprazole in schizophrenia with PET and [(18)F]Fallypride. Neuropsychopharmacology 2008;33:3111-3125.

42. Catafau AM, Tolosa E. Impact of dopamine transporter SPECT using 123I-Ioflupane on diagnosis and management of patients with clinically uncertain Parkinsonian syndromes. Mov Disord 2004;19:1175-1182.

43. Abi-Dargham A, Rodenhiser J, Printz D, et al. Increased baseline occupancy of D2 receptors by dopamine in schizophrenia. Proc Natl Acad Sci USA 2000;97:8104-8109.

44. Abi-Dargham A, Giessen EV, Slifstein M, Kegeles LS, Laruelle M. Baseline and amphetamine-stimulated dopamine activity are related in drug-naive schizophrenic subjects. Biol Psychiatry 2009;65: 1091-1093.

45. Woodward ND, Zald DH, Ding Z, et al. Cerebral morphology and dopamine D2/D3 receptor distribution in humans: a combined [18F]fallypride and voxel-based morphometry study. Neuroimage 2009;46:31-38.

46. Buchsbaum MS, Christian BT, Lehrer DS, et al. D2/D3 dopamine receptor binding with $[\mathrm{F}-18]$ fallypride in thalamus and cortex of patients with schizophrenia. Schizophr Res 2006;85:232-244.

47. Catafau AM, Perez V, Plaza P, et al. Serotonin transporter occupancy induced by paroxetine in patients with major depression disorder: a 123I-ADAM SPECT study. Psychopharmacology (Berl) 2006;189:145-153.

48. Pissarek MB, Oros-Peusquens AM, Schramm NU. Challenge by the murine brain: multi-pinhole SPECT of 123I-labelled pharmaceuticals. J Neurosci Methods 2008;168:282-292.

49. Yokoi F, Grunder G, Biziere K, et al. Dopamine D2 and D3 receptor occupancy in normal humans treated with the antipsychotic drug aripiprazole (OPC 14597): a study using positron emission tomography and $[11 \mathrm{C}]$ raclopride. Neuropsychopharmacology 2002;27:248-259.

50. Suhara T, Takano A, Sudo Y, et al. High levels of serotonin transporter occupancy with low-dose clomipramine in comparative occupancy study with fluvoxamine using positron emission tomography. Arch Gen Psychiatry 2003;60:386-391.

51. Baumann P, Hiemke C, Ulrich S, et al. The AGNP-TDM expert group consensus guidelines: therapeutic drug monitoring in psychiatry. Pharmacopsychiatry 2004;37:243-265.

52. Keller M, Montgomery S, Ball W, et al. Lack of efficacy of the substance $\mathrm{p}$ (neurokinin1 receptor) antagonist aprepitant in the treatment of major depressive disorder. Biol Psychiatry 2006;59:216-223.

53. Harmer CJ, Mackay CE, Reid CB, Cowen PJ, Goodwin GM. Antidepressant drug treatment modifies the neural processing of nonconscious threat cues. Biol Psychiatry 2006;59:816-820.

54. Yoon SJ, Lyoo IK, Haws C, Kim TS, Cohen BM, Renshaw PF Decreased glutamate/glutamine levels may mediate cytidine's efficacy in treating bipolar depression: a longitudinal proton magnetic resonance spectroscopy study. Neuropsychopharmacology 2009;34:1810-1818.

55. Kozel FA, Johnson KA, Nahas Z, et al. Fractional anisotropy changes after several weeks of daily left high-frequency repetitive transcranial magnetic stimulation of the prefrontal cortex to treat major depression. J ECT 2010 Oct 1. [Epub ahead of print].

56. Coenen VA, Honey CR, Hurwitz T, et al. Medial forebrain bundle stimulation as a pathophysiological mechanism for hypomania in subthalamic nucleus deep brain stimulation for Parkinson's disease. Neurosurgery 2009;64:1106-1114; discussion 11141105.

57. Masdeu J. Neuroimaging of disorders leading to dementia. Continuum (AAN) 2008;14:144-163. 